\title{
New Records for GASTROPODA Class of Species Found in the Rocky Intertidal Zone of the Marine Priority Region 32, Guerrero, Mexico
}

\author{
Carmina Torreblanca-Ramírez1, Rafael Flores-Garza², Jesús Emilio Michel-Morfin³, \\ José Luis Rosas-Acevedo', Pedro Flores-Rodríguez², Sergio García-Ibáñez ${ }^{2}$ \\ ${ }^{1}$ Unidad de Ciencias de Desarrollo Regional, Universidad Autónoma de Guerrero, Acapulco, Mexico \\ ${ }^{2}$ Laboratorio de Ecología Costera y Sustentabilidad, Unidad Académica de Ecología Marina, Universidad \\ Autónoma de Guerrero, Acapulco, Mexico \\ ${ }^{3}$ Departamento de Estudios para el Desarrollo Sustentable de Zonas Costeras, Universidad de Guadalajara, \\ Melaque, Mexico \\ Email: carminatorreblanca@yahoo.com.mx, rfloresgarza@yahoo.com, michel11012@yahoo.com.mx
}

Received 15 May 2014; revised 22 June 2014; accepted 3 July 2014

Copyright (C) 2014 by authors and Scientific Research Publishing Inc.

This work is licensed under the Creative Commons Attribution International License (CC BY). http://creativecommons.org/licenses/by/4.0/

(c) (i) Open Access

\section{Abstract}

The GASTROPODA class, one of the best known marine environments, is formed by a large number of species and the shape of their shell structure varies greatly. These mollusks are distributed from the intertidal zone all the way to the abyssal zone, and there are also swimming and floating species. This research was conducted at seven sites, located in the Marina Priority Region 32 (MPR 32), located in the State of Guerrero, Mexico. The aim of this report is to document the scope of the geographic distribution of the 34 species of GASTROPODA class. 11,263 total specimens were analyzed. 108 species were identified, of which four species are new records for Mexico, 15 for the Transitional Mexican Pacific, 11 for the State of Guerrero and four for the MPR 32. Here we report a significant number of new records of species of class GASTROPODA found on the rocky intertidal zone of the MPR 32. The reporting of these new records, demonstrates the need to focus research efforts on the study of marine diversity, since knowledge in this respect is quite incomplete, especially regarding marine mollusks, a fact that has been reported by the National Commission for the Management and Use of Biodiversity.

\section{Keywords}

GASTROPODA, Biodiversity, New Records, Intertidal Zone and Guerrero 


\section{Introduction}

Mexico has seven of the 24 marine ecoregions into which North America is divided. Region 17, also called the Mexican Pacific Transitional includes the coastal waters of the states of Jalisco, Colima, Michoacán, Guerrero and Oaxaca and the southern tip of Baja California Sur. The physiography of this region is characterized by a narrow continental shelf with an amplitude of $10-15 \mathrm{~km}$ and a slope of less than $1^{\circ} 30^{\prime}$, with a deep ocean trench and complex abyssal plains [1].

For the State of Guerrero the National Commission for the Knowledge and Use of Biodiversity (Comision Nacional Para el Uso y Conocimiento de la Biodiversidad; CONABIO) stated that there are four marine priority areas for the conservation of coastal and ocean biodiversity; about these regions CONABIO has highlighted the lack of knowledge about the diversity of marine species.

The Marine Priority Region No. 32 (MPR 32) is located in the state of Guerrero and is known by the name Coyuca-Tres Palos, has an area of $829 \mathrm{~km}^{2}$. This region is identified as an area of high biodiversity and that presents problems as reported by CONABIO due to the modification of the environment by discharges of fresh water, chemicals and fertilizers, live stock waste, damage to the environment by tourism, the use of its resources such as endangered bird species, the introduction of exotic species such as tilapia and coconut palm, and the lack of current regulations for the use of natural resources [2].

As part of the marine fauna that make up the MPR 32 are mollusks, and within this taxon is the GASTROPODA class, which are known as the best of the marine environment, being numerous and varied as to the form of their shells

These organisms have a muscular foot retractor having the function of moving the head and foot of the mollusk in the shell. They are distributed from the intertidal zone to the deep or abyssal zone, but there are also swimming and floating species [3].

For organisms that belong to the class GASTROPODA, there are reports concerning global taxonomic and geographical aspects [4] [5]. There are also reports of this type exclusive to the American Pacific as [6]-[8].

In the Mexican Pacific research has been conducted involving taxonomic aspects, composition and structure of communities; others include diversity, abundances, zonation, variation over time and geographical distribution of species, such as [9]-[24].

In particular for the coasts of Guerrero where there are reports of the class GASTROPODA analyzed as part of the set of classes studied, among which are those of [25]-[37], however, the class GASTROPODA in the coast of Guerrero, there are still many outstanding issues, regarding the knowledge of biodiversity. It is important to have an inventory of species, to study communities and composition, and to manage and maintain biodiversity of highly diverse regions such as the state of Guerrero in rational and responsible manner.

The aim of this contribution is to report the scope of geographical distribution for 34 species of the class GASTROPODA found in MPR 32.

\section{Materials and Methods}

\section{Study Area}

The coast of Guerrero is located in the 17th Marine Ecoregion of North America. As part of the coast of Guerrero is the MPR 32, (Coyuca-Tres Palos area) is located at latitude $16^{\circ} 35^{\prime} 24^{\prime \prime}$ to $17^{\circ} 28^{\prime} 12^{\prime \prime}$ and longitude $99^{\circ} 25^{\prime} 12^{\prime \prime}$ to $100^{\circ} 33^{\prime}$ (Figure 1).

The climate is warm sub-humid, has an average annual temperature over $26^{\circ} \mathrm{C}$, tropical storms and hurricanes occur from May to November. It belongs to the North American plate, is constituted by metamorphic rocks and a narrow platform. It is bathed by Costa Rica Coastal and North Equatorial Currents, receives fresh-water input from rivers, some areas are hit by harsh high surf waves, el Niño, red tide and other processes of turbulence occurs. It is an area with a rich and abundant biodiversity: mollusks, polychaetes, echinoderms, crustaceans, turtles, fish, birds, marine mammals, endemic fish and mangrove, and is an important bird feeding area. There are also regulatory issues; ignorance of current regulations for the use of resources and illegal fishing is frequent. This region is particularly important because it represents an area for several zoological groups with respect to conservation, especially birds and their diversified habitat, and the potential impact by tourism and the lack of information on this issue [2].

Sampling was conducted from 2009 to 2012 at seven sites: Parque de la Reina, Tlacopanocha, Majahua, 


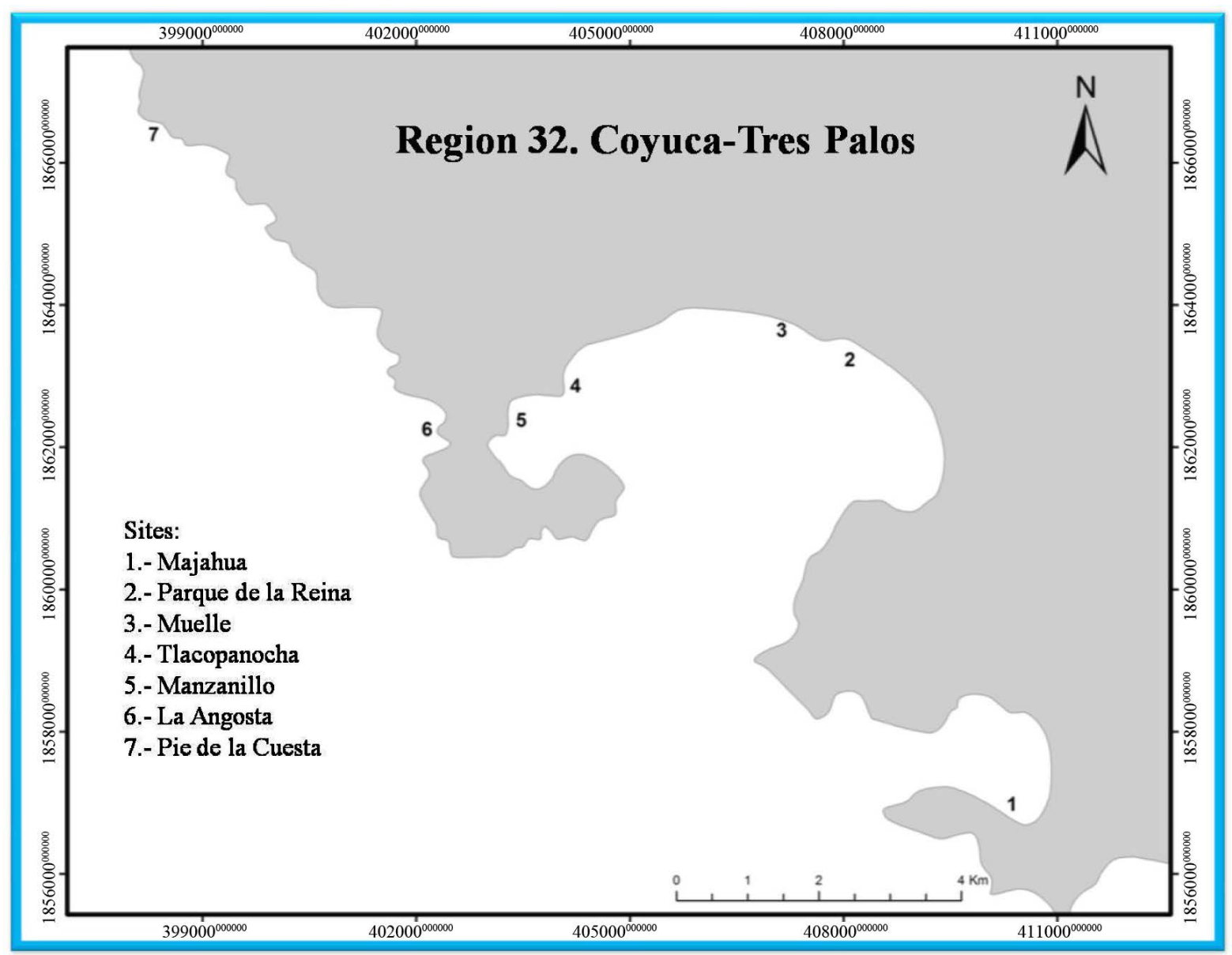

Figure 1. Marine Priority Regions in the state of Guerrero, Mexico and sampling sites. Source: National Commission for the Knowledge and Use of Biodiversity (CONABIO) Authors: C. Torreblanca-Ramírez, R. Flores-Garza and L. Galiana-Rebolledo.

Muelle, Manzanillo, La Angosta and Pie de la Cuesta. Geographical coordinates were registered for each site and described according to the following criteria: approximate length of the sampling area, the substrate structure and stability, rock type, and wave exposure of the substrate. Descriptions of the collection sites were based on [38], geological maps of the National Institute of Statistics, Geography and Informatics "Instituto Nacional de Estadística, Geografía e Informática” INEGI (Acapulco maps E14-11, 1:50,000), and complemented with field observations (Table 1).

The sites may change with the type of substrate, its stability and exposure to wave impact; and were classified as substrate Type: 1) Rock masses: fixed structures such as walls, cliffs, terraces, and alike. 2) Large boulders: unattached rocks larger than $50 \mathrm{~cm}$, immovable or difficult to move by the waves impact. 3) Rolled boulders: loose rock smaller than $50 \mathrm{~cm}$ and greater than $8 \mathrm{~cm}$. that can easily be moved by the impact of the waves. 4) Gravel: loose rocks o pebbles no larger than $8 \mathrm{~cm}$. The substrate stability was classified as: 1) High: when the substrate remains practically unchanged by the impact of waves, 2) Middle: when the configuration of the substrate is basically not changed by the waves impact but there is some rock displacement, 3) Low: when the site configuration is changed by the impact of waves, most of the rocks are moved. Wave exposure was classified as: 1) High: when waves hits the unprotected substrate directly, 2) Middle: when the wave impact on the substrate is softened or hampered by barriers and 3) Low: when the wave does not hit directly, since the sites are protected by different types of barriers [39].

\section{Collection of Samples}

Three samples were conducted per site between 2009. Sampling was performed during the hours of low tide on 
Table 1. Sampling sites considered in the study, indicating the location and relevant characteristics of the habitat.

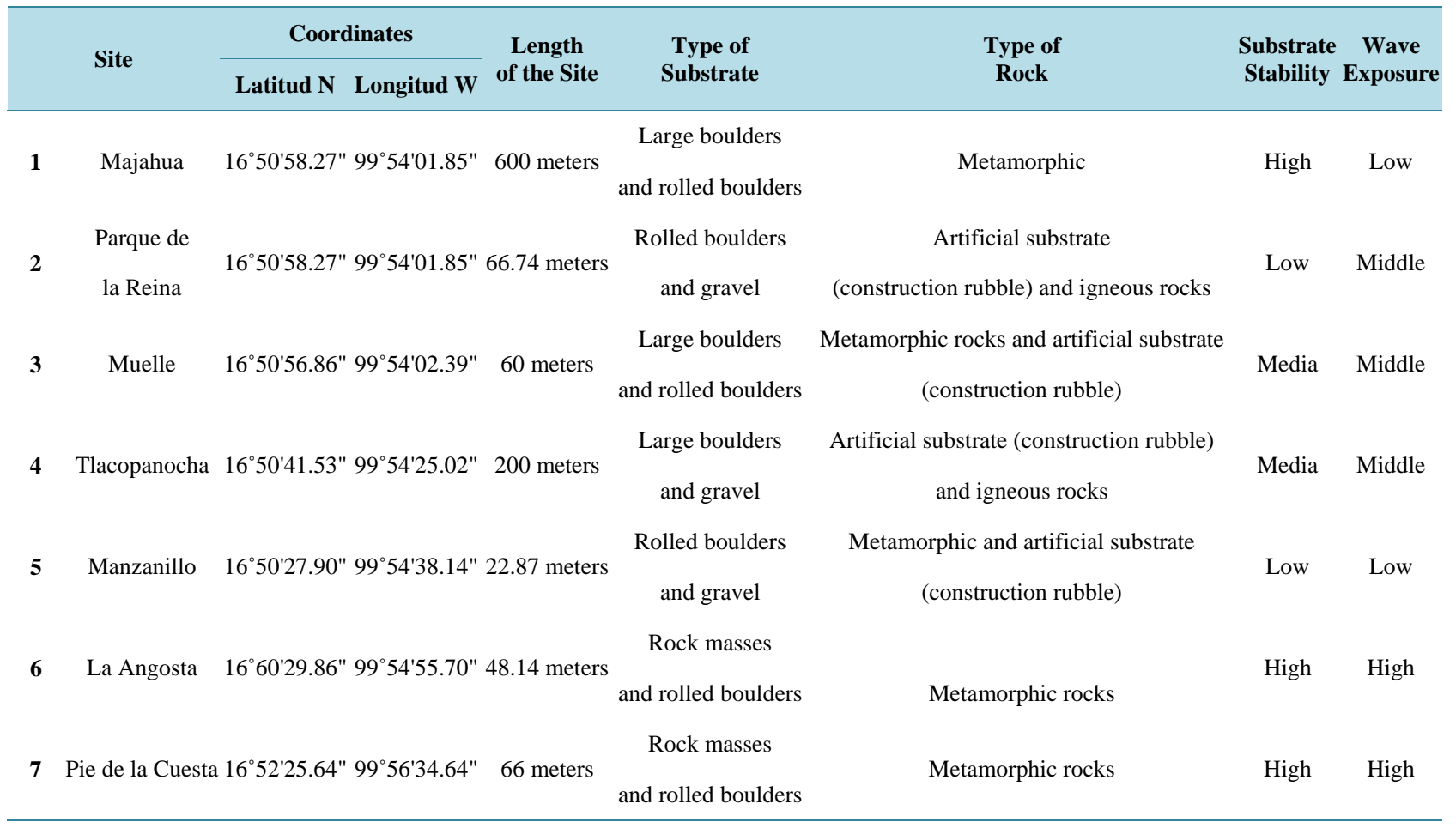

new moon days. Sampling was carried out using quadrants and travels in search of organisms that were not found in the quadrants. The quadrants were used for the purpose of carefully reviewing an area and to keep smaller organisms from being excluded from the sample and this was carried out in the following way. The starting point was randomly selected, then a nylon rope $30 \mathrm{~m}$ long was placed parallel to the shore on the intertidal zone using a PVC pipe square frame $1 \mathrm{~m}$ per side to delimit the sampling unit. Once the starting point was established, the PVC frame was placed at the selected point. All GASTROPODA specimens found alive, even those attached under rocks within the sampling unit, were collected and placed into a plastic container filled with seawater and protected from direct sunlight. After completing the collection, a 2-m section along the rope was measured then the frame was again placed to define the following sampling unit. This procedure was repeated ten times along the length of the nylon rope.

Upon completion of the sampling there was a preservation of the specimens where there was doubt about its identification. The specimens were placed in jars with $96 \%$ ethyl alcohol and were transferred to the laboratory.

\section{Laboratory Work and Data Analysis}

The collected specimens were identified and quantified, using literature [5] and the nomenclature was updated according to [8]. Literature and specialized websites [40]-[42] were consulted to determine if the organisms identified in the field and returned alive to their habitat and collected were recorded in the Pacific Coast of Mexico and particularly in the MPR 32.

The collected specimens were measured in length and width and the values of descriptive statistics such as maximum, minimum, average and standard deviation were obtained, these values are expressed in millimeters and were obtained using a digital caliper.

Subsequently, the specimens were deposited in the National Collection of Molluscs at the National Institute of Biology, Universidad Nacional Autónoma de México and the Molluscs Colletion of the Academic Unit of Marine Ecology of the Universidad Autónoma de Guerrero

\section{Results}

35 species were identified as new records. Four species are new records for Mexico (Table 2 and Figure 2), 15 


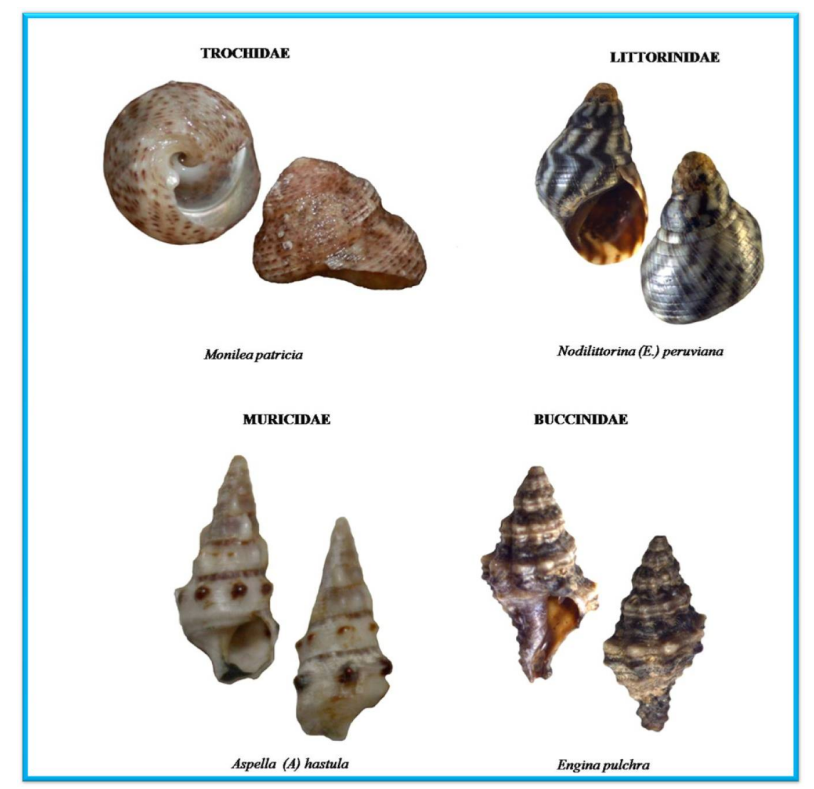

Figure 2. New records for Mexico. Pictures collection of marine invertebrates, Unidad Académica de Ecología Marina, Universidad Autónoma de Guerrero. Authors: C. TorreblancaRamírez, R. Flores-Garza, L. Galeana-Rebolledo y J.C. Cerros-Cornelio.

Table 2. New records for Mexico, examined material, sizes and distributions prior to this contribution.

\begin{tabular}{|c|c|c|c|c|}
\hline Family/species & $\begin{array}{l}\text { Revised } \\
\text { number of } \\
\text { specimens }\end{array}$ & Long sizes (mm) & Known distribution & New distribution \\
\hline $\begin{array}{c}\text { TROCHIDAE } \\
\text { Rafinesque, } 1815 .\end{array}$ & & Mx. Min. A. Sd. & & \\
\hline $\begin{array}{l}\text { Monilea patricia } \\
\text { (Philippi, 1851). }\end{array}$ & 12 & 10.334 .217 .051 .98 & $\begin{array}{l}\text { West America to Nicaragua (Corintos) and } \\
\text { Colombia. (A. Myra Keen 1971, WoRMS: } \\
\text { World Register of Marine Species 2013 and WMSDB: } \\
\text { Worldwide Mollusc Species Data Base 2013.) }\end{array}$ & $\begin{array}{c}\text { Majahua } \\
16^{\circ} 47^{\prime} 39.62^{\prime \prime} \mathrm{N} \mathrm{y} 99^{\circ} 50^{\prime} 29.02^{\prime \prime} \mathrm{W} \\
\text { Tlacopanocha } \\
16^{\circ} 50^{\prime} 41.53^{\prime \prime} \mathrm{N} \text { y } 99^{\circ} 54^{\prime} 25.02^{\prime \prime} \mathrm{W}\end{array}$ \\
\hline \multicolumn{5}{|l|}{$\begin{array}{l}\text { LITTORINIDAE } \\
\text { Children, 1834. }\end{array}$} \\
\hline $\begin{array}{c}\text { Nodilittorina } \\
\text { (Echinolittorina) } \\
\text { peruviana } \\
\text { (Lamarck, 1822). }\end{array}$ & 3 & $6.41 \quad 6.16 .290 .16$ & $\begin{array}{l}\text { Central America, Costa Rica, Panama (Western Panama, } \\
\text { Chiriqui Bay), Ecuador (Galapagos Islands), Peru } \\
\text { (north of Punta Peña Negra, Talara) and Chile (Isla } \\
\text { Serrano). (A. Myra Keen 1971, Carol Skoglund 2002, } \\
\text { WoRMS: World Register of Marine Species } 2013 \text { and } \\
\text { WMSDB: Worldwide Mollusc Species Data Base 2013.) }\end{array}$ & $\begin{array}{c}\text { Parque de la Reina } \\
16^{\circ} 50^{\prime} 58.27^{\prime \prime N} \text { y } 99^{\circ} 54^{\prime} 01.85^{\prime \prime W}\end{array}$ \\
\hline \multicolumn{5}{|l|}{$\begin{array}{l}\text { MURICIDAE } \\
\text { Follows Vokes, } \\
1996 .\end{array}$} \\
\hline $\begin{array}{l}\text { Aspella } \\
\text { Aspella) hastula } \\
\text { (Reeve, 1844). }\end{array}$ & 1 & & $\begin{array}{l}\text { Costa Rica (Cocos Island), Panama (Gulf of Chiriqui), } \\
\text { Ecuador (Galapagos Islands) and Colombia (Malpelo } \\
\text { Island). (A. Myra Keen 1971, Carol Skoglund 2002, } \\
\text { WMSDB: Worldwide Mollusc Species Data Base 2013 } \\
\text { and Discover Life 2013.) }\end{array}$ & $\begin{array}{c}\text { Tlacopanocha } \\
16^{\circ} 50^{\prime} 41.53^{\prime \prime N} \text { y } 99^{\circ} 54^{\prime} 25.02 " \mathrm{~W}\end{array}$ \\
\hline \multicolumn{5}{|l|}{$\begin{array}{c}\text { BUCCINIDAE } \\
\text { Rafinesque, } 1815 .\end{array}$} \\
\hline $\begin{array}{l}\text { Engina pulchra } \\
\text { (Reeve, 1846). }\end{array}$ & 1 & 6.456 .456 .45 & $\begin{array}{l}\text { North of El Salvador, Panama (Fortkobbe beach), } \\
\text { Colombia (Gorgona Island) and Ecuador } \\
\text { (Galapagos Islands) (A. MyraKeen 1971, Carol Skoglund } \\
2002 \text { and Discover Life 2013.) }\end{array}$ & $\begin{array}{c}\text { Muelle } \\
16^{\circ} 50^{\prime} 58.27^{\prime \prime} \mathrm{N} \mathrm{y} 99^{\circ} 54^{\prime} 01.85^{\prime} \mathrm{W}\end{array}$ \\
\hline
\end{tabular}

Mx.: Maximum, Min.: Minimum, A.: Average and Sd.: Standard deviation. 
are new records for the Transitional Mexican Pacific (Table 3 and Figure 3), 11 are new records for the state of Guerrero (Table 4 and Figure 4) and seven are new records for the Marine Priority Region 32 (Table 5 and Figure 5).

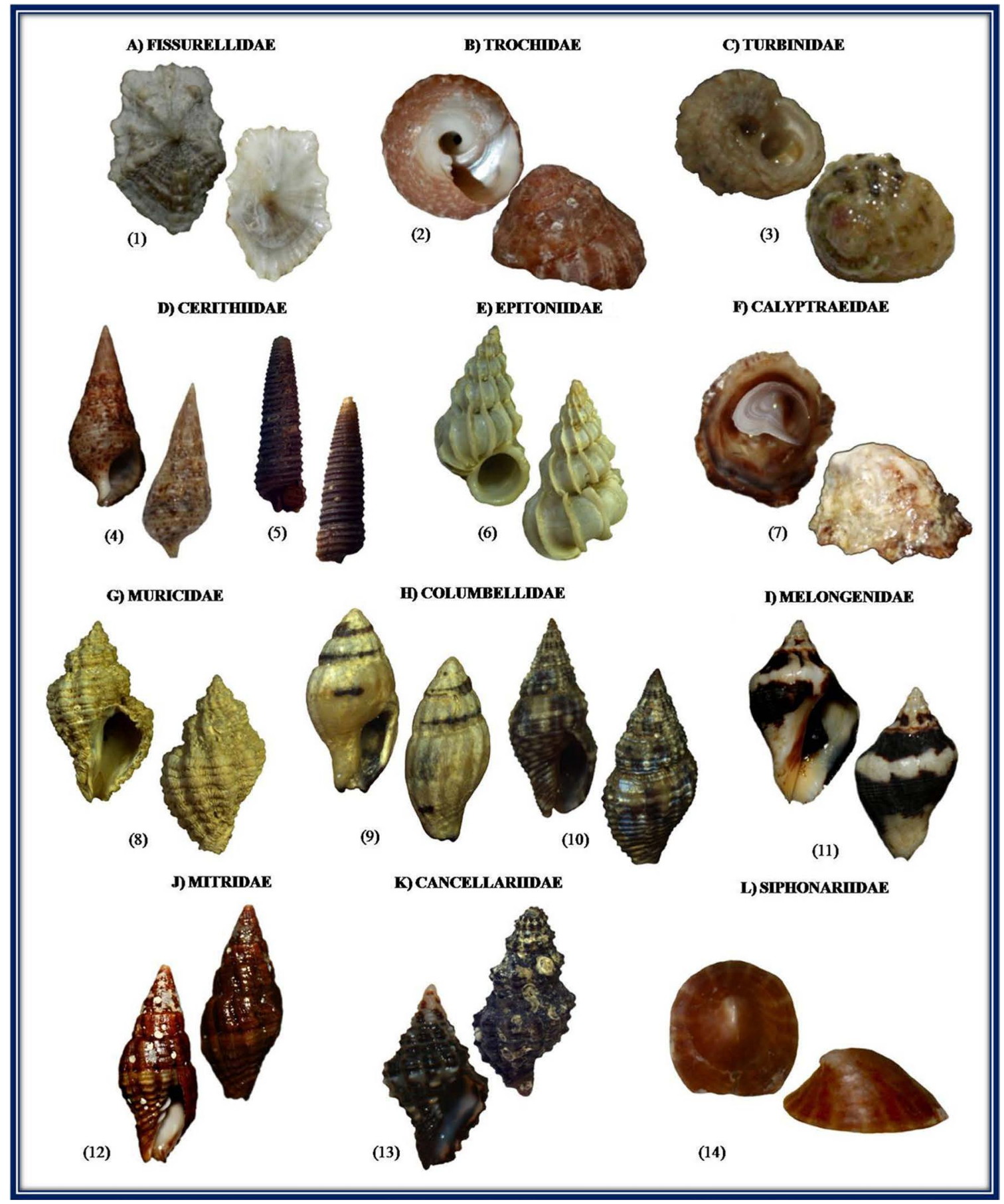

Figure 3. New records for Transitional Mexican Pacific. 1 A) Hemitoma (H.) natlandi, 2 B) Tegula (A.) panamensis, 3 C) Arene (A.) hindsiana, 4 D) Rhinoclavis (O.) gemmata, 5 D) Seila assimilata, 6 E) Epitonium (L.) cookeanum, 7 F) Crucibulum (D.) subactum, 8 G) Coralliophila (P.) parva, 9 H) Parvanachis dalli, 10 H) Decipifus lyrta, 11 I) Melongena patula, $12 \mathrm{~J}$ ) Mitra lignaria, $13 \mathrm{~K}$ ) Cancellaria (S.) buccinoides and $14 \mathrm{~L}$ ) Williamia peltoide. Pictures collection of marine invertebrates, Unidad Académica de Ecología Marina, Universidad Autónoma de Guerrero. Authors: C. Torreblanca-Ramírez, R. Flores-Garza, L. Galeana-Rebolledo y J.C. Cerros-Cornelio. 
Table 3. New records for Transitional Mexican Pacific, examined material, sizes and distributions prior to this contribution.

\begin{tabular}{|c|c|c|c|c|}
\hline Family/species & $\begin{array}{c}\text { Revised } \\
\text { number of } \\
\text { specimens }\end{array}$ & Long sizes (mm) & Known distribution & New distribution \\
\hline
\end{tabular}

FISSURELLIDAE

Fleming, C.A., 1822.

Hemitoma

(Hemitoma)

natlandi

Durham, 1950.

\section{TROCHIDAE}

Tegula

(Agathistoma)

panamensis

(Philippi, 1849).

TURBINIDAE

Rafinesque, 1815.

\author{
Arene (Arene) \\ hindsiana
}

Pilsbry\& Lowe, 1932.

\section{CERITHIIDAE}

\section{Fleming, 1822.}

\author{
Rhinoclavis \\ (Ochetoclava) \\ gemmata
}

(Hinds, 1844).

\section{Seila assimilata}

(C. B. Adams, 1852)
Mx. Min. A. Sd.

$\begin{array}{lll}15.17 & 15.17 & 15.17\end{array}$
Mexico (Barra de Navidad, Jalisco), Panama (Panama Bay) and Colombia (Puerto Utria and Gorgona Island)

(A. Myra Keen 1971, Carol Skoglund 2002, WoRMS: World Register of Marine Species 2013 and Discover Life 2013).
Majahua $16^{\circ} 47 ' 39.62 " \mathrm{~N} \mathrm{y}$ $99^{\circ} 50 ' 29.02^{\prime \prime W}$

\section{$\begin{array}{lllll}3 & 9.38 & 4.37 & 6.87 & 3.54\end{array}$}

(n)

\section{Mexico (Sonora), El Salvador (Playa La Libertad), \\ Costa Rica (Del Coco and Jaco beaches), Panama (Venado Island), Ecuador, Colombia} (north of the island of Gorgona) and Peru (south of Paita) (A. Myra Keen 1971, Carol Skoglund 2002, WoRMS: World Register of Marine Species 2013, WMSDB: Worldwide Mollusc Species Data Base 2013 and Discover Life 2013).

\author{
Majahua \\ $16^{\circ} 47^{\prime} 39.62^{\prime \prime} \mathrm{N} \mathrm{y}$ \\ $99^{\circ} 50$ '29.02"W \\ Manzanillo \\ $16^{\circ} 50^{\prime} 27.90^{\prime \prime} \mathrm{N} \mathrm{y}$ \\ $9^{\circ} 54$ '38.14"W
}

American West, Mexico (Baja California Sur, Colima Manzanillo, Tres Marias Islands, Mazatlan and Manzanillo.) (A. Myra Keen 1971, WMSDB: Worldwide Mollusc Species Data Base 2013 and Discover Life 2013).
Majahua $16^{\circ} 47^{\prime} 39.62^{\prime \prime} \mathrm{N} \mathrm{y}$ $99^{\circ} 50$ '29.02"W Tlacopanocha $16^{\circ} 50^{\prime} 41.53 " \mathrm{~N} \mathrm{y}$ $99^{\circ} 54$ '25.02"W
Mexico (Magdalena Bay to the Gulf of California, Baja California Sur, Revillagigedo Island, Manzanillo, Colima;) Colombia (Gorgona Island) and Ecuador (GalapagosIslands)

(A. Myra Keen 1971 and Carol Skoglund 2002).

EE. UU. (Todas Santos Bay, California), Mexico (Puertecitos Baja California, Gulf of California, Todas Santos Bay, Bahia Magdalena and San Felipe, Baja California Sur and La Cruz, Nayarit), Costa Rica (Cocos Island) Panama (Panama Bay), Colombia (Malpelo Island and Gorgona Island) Ecuador (Galapagos Islands) and Peru (south of Tumbes). (A. Myra Keen 1971, WoRMS: World Register of Marine Species 2013 and Discover Life 2013).

\section{EPITONIIDAE} Berry 1910.
Epitonium
(Labeoscala)
cookeanum
Dall, 1917.

EE. UU. (San Diego, California), Mexico (California Gulf, La Paz, Cabo San Lucas, Isla de Espiritu Santo, Baja California Sur. Peñasco Bay, Guaymas Sonora) west of Panama (A. Myra Keen 1971, Carol Skoglund 2002, WoRMS: World Register of Marine Species 2013 and Discover Life 2013).

\author{
Parque de la \\ Reina \\ $16^{\circ} 50^{\prime} 58.27 " \mathrm{~N} \mathrm{y}$ \\ $99^{\circ} 54^{\prime} 01.85 " \mathrm{~W}$
}




\section{Continued}

\section{CALYPTRAEIDAE \\ Lamarck, 1809.}

Crucibulum

(Dispotaea) subactum

Berry, 1963.
$29.24 \quad 7.25 \quad 11.68 \quad 6.86$

American West, Mexico (Gulf of California, Santa Rosalia, Baja California Sur. Guaymas, Sonora and Sinaloa) and Ecuador (south of Manabi Province)

(A. Myra Keen 1971, Carol Skoglund 2002, WMSDB: Worldwide Mollusc Species Data Base 2013 and Discover Life 2013).

Parque de la Reina

$16^{\circ} 50^{\prime} 58.27 " \mathrm{~N}$ y

$99^{\circ} 54^{\prime} 01.85^{\prime \prime} \mathrm{W}$

Muelle

$16^{\circ} 50^{\prime} 58.27 " \mathrm{~N} \mathrm{y}$ $99^{\circ} 54^{\prime} 01.85 " \mathrm{~W}$

Tlacopanocha

$16^{\circ} 50^{\prime} 41.53 " \mathrm{~N} \mathrm{y}$

$99^{\circ} 54^{\prime 2} 25.02^{\prime \prime} \mathrm{W}$

Pie de la Cuesta

$16^{\circ} 52$ '25.64"N y $99^{\circ} 56 ' 34.64 " \mathrm{~W}$

\section{MURICIDAE}

Coralliophila

$\begin{array}{lllll}\text { (Pseudomurex) parva } & 1 & 9.05 & 9.05 & 9.05\end{array}$

(E. A. Smith, 1877)

\section{COLUMBELLIDAE}

Swainson, 1440.
Mexico (Puertecitos, Baja California, Gulf of California, La Paz, Baja California Sur, Revillagigedo Island, Bay of Santo Domingo, Manzanillo, Colima); Clipperton Island; Costa Rica (Cocos Island); Panama (Panama Bay and Deer Island); Colombia (Malpelo Island) and Ecuador (Galapagos Islands)

(A. Myra Keen 1971, Carol Skoglund 2002, WoRMS: World Register of Marine Species 2013, WMSDB: Worldwide Mollusc Species Data Base 2013 and Discover Life 2013).
Parque de la Reina $16^{\circ} 50^{\prime} 58.27 " \mathrm{~N} \mathrm{y}$ $99^{\circ} 54^{\prime} 01.85^{\prime \prime} \mathrm{W}$
Mexico (Cuastecomate Bay, Jalisco), Panama (Panama Bay and Tortola Island), Colombia (Malpelo Island), Ecuador (Punta Blanco) and southern Peru (Tumbes);

(A. Myra Keen 1971, Carol Skoglund 2002, WMSDB: Worldwide Mollusc Species Data Base 2013 and Discover Life 2013).
Decipifus lyrta

(Baker, Hanna \& Strong, 1938)

$\begin{array}{ccccc}9 & 8.77 & 7.3 & 7.9 & 0.4 \\ & & 4 & 5 & 9\end{array}$

\begin{abstract}
West Part of America, Mexico (Northern Gulf of California to San Luis Gonzaga Bay, Port of San Felipe and Isla Angel de la Guarda,
\end{abstract}

Baja California Sur and Mazatlan, Sinaloa)

(A. Myra Keen 1971, WMSDB: Worldwide Mollusc Species Data Base 2013 and Discover Life 2013).
Majahua

$16^{\circ} 47^{\prime} 39.62^{\prime \prime} \mathrm{N} \mathrm{y}$ $99^{\circ} 50^{\prime} 29.02^{\prime \prime} \mathrm{W}$

Parque de la Reina $16^{\circ} 50^{\prime} 58.27 " \mathrm{~N} \mathrm{y}$ $99^{\circ} 54^{\prime} 01.85 " \mathrm{~W}$

Muelle $16^{\circ} 50^{\prime} 58.27 " \mathrm{~N} \mathrm{y}$ $99^{\circ} 54^{\prime} 01.85 " \mathrm{~W}$

Tlacopanocha

$16^{\circ} 50^{\prime} 41.53 " \mathrm{~N}$ y $99^{\circ} 54^{\prime 25.02 " \mathrm{~W}}$

Manzanillo $16^{\circ} 50^{\prime} 27.90^{\prime \prime} \mathrm{N} \mathrm{y}$ $99^{\circ} 54^{\prime} 38.14 " \mathrm{~W}$ Pie de la Cuesta $16^{\circ} 52^{\prime} 25.64 " \mathrm{~N}$ y $99^{\circ} 56 ' 34.64 " \mathrm{~W}$

Majahua $16^{\circ} 47^{\prime} 39.62^{\prime \prime} \mathrm{N} \mathrm{y}$ 99 50'29.02"W

Parque de la Reina $16^{\circ} 50^{\prime} 58.27 \mathrm{~N} \mathrm{y}$ $99^{\circ} 54^{\prime} 01.85^{\prime \prime} \mathrm{W}$

Tlacopanocha $99^{\circ} 54^{\prime} 25.02 " \mathrm{~W}$ 


\section{Continued}

Steironepion

$\begin{array}{lllll}\text { melanosticta } & 1 & 4.57 & 4.57 & 4.57\end{array}$

Pilsbry \& Lowe, 1832.

\section{MELONGENIDAE}

Gill, 1871.

Melongena patula

(Broderip \& Sowerby 1829)

Mitra lignaria

Reeve, 1844. $\begin{array}{lllll}10 & 30.11 & 25.85 & 27.71 & 1.481\end{array}$ (1)
Tlacopanocha

$16^{\circ} 50^{\prime} 41.53^{\prime \prime} \mathrm{N} \mathrm{y}$ $99^{\circ} 54^{\prime} 25.02 " \mathrm{~W}$

Province of Manabí and the Galapagos Islands)

(A. Myra Keen 1971, Carol Skoglund 2002, and Discover Life 2013).

Mexico (Playa Estero, Ensenada, Baja California, Gulf of California, Mar de Cortez, Guaymas, Sonora and Barra de Navidad, Jalisco) west of Guatemala, Honduras (Balfate), Costas Rica, Panama (West of Panama in San Blas and Venado Island), Ecuador (Esmeralda) and Peru (Tumbes and Puerto Pizarro)

(A. Myra Keen 1971, Carol Skoglund 2002, WMSDB: Worldwide Mollusc Species Data Base 2013 and Discover Life 2013).
Tlacopanocha $16^{\circ} 50^{\prime} 41.53 " \mathrm{~N} \mathrm{y}$ $99^{\circ} 54^{\prime} 25.02 \mathrm{WW}$

Manzanillo $16^{\circ} 50 ' 27.90 " \mathrm{~N} \mathrm{y}$ 9954'38.14"W

Angosta

$16^{\circ} 60^{\prime} 29.86^{\prime \prime} \mathrm{N} \mathrm{y}$ $99^{\circ} 54^{\prime} 5.70^{\prime \prime} \mathrm{W}$

Majahua
Mexico (Guaymas, Sonora y Mazatlán, Sinaloa) a Panamá; Colombia and Ecuador.

(A. Myra Keen 1971, WoRMS: World Register of Marine Species 2013, WMSDB:

Worldwide Mollusc Species Data Base 2013 and Discover Life 2013).

$16^{\circ} 47^{\prime} 39.62 " \mathrm{~N} \mathrm{y}$ $99^{\circ} 50^{\prime} 29.02 " \mathrm{~W}$

Tlacopanocha $16^{\circ} 50^{\prime} 41.53 " \mathrm{~N} \mathrm{y}$ $99^{\circ} 54^{\prime} 25.02^{\prime \prime} \mathrm{W}$

Manzanillo

$16^{\circ} 50^{\prime} 27.90^{\prime \prime} \mathrm{N} \mathrm{y}$ $99^{\circ} 54 ' 38.14 " \mathrm{~W}$

Majahua

Mexico (Magdalena Bay, Baja California Sur), Costa Rica (Puntaneras), Nicaragua, Chile, Peru (Pampa de Palo, Moquegua) and Chile (Iglesia Bay and Tongoy)

(A. Myra Keen 1971 y Discover Life 2013). $16^{\circ} 47^{\prime} 39.62 " \mathrm{~N} \mathrm{y}$ $99^{\circ} 50 ' 29.02^{\prime \prime} \mathrm{W}$

Muelle $16^{\circ} 50^{\prime} 58.27 " \mathrm{~N} \mathrm{y}$ $99^{\circ} 54^{\prime} 01.85^{\prime \prime W}$

\section{SIPHONARIIDAE}

Gray, 1827.

Williamia peltoides

(Carpenter, 1864).
$1 \quad 5.51 \quad 5.51 \quad 5.51$
North of EE. UU. (Crescent, California), Mexico

(Southern California to the Gulf of California, Rocas Alijos Island, Revillagigedo Island, Manzanillo, Colima), Costa Rica (Cocos Island),

North and South Panamic Province, Colombia (Malpelo Island) and Ecuador (Galapagos Islands).

(A. Myra Keen 1971, Carol Skoglund 2002, WoRMS: World Register of Marine Species 2013 and WMSDB: Worldwide Mollusc Species Data Base 2013). 
Table 4. New records for the State of Guerrero, México, examined material, sizes and distributions prior to this contribution.

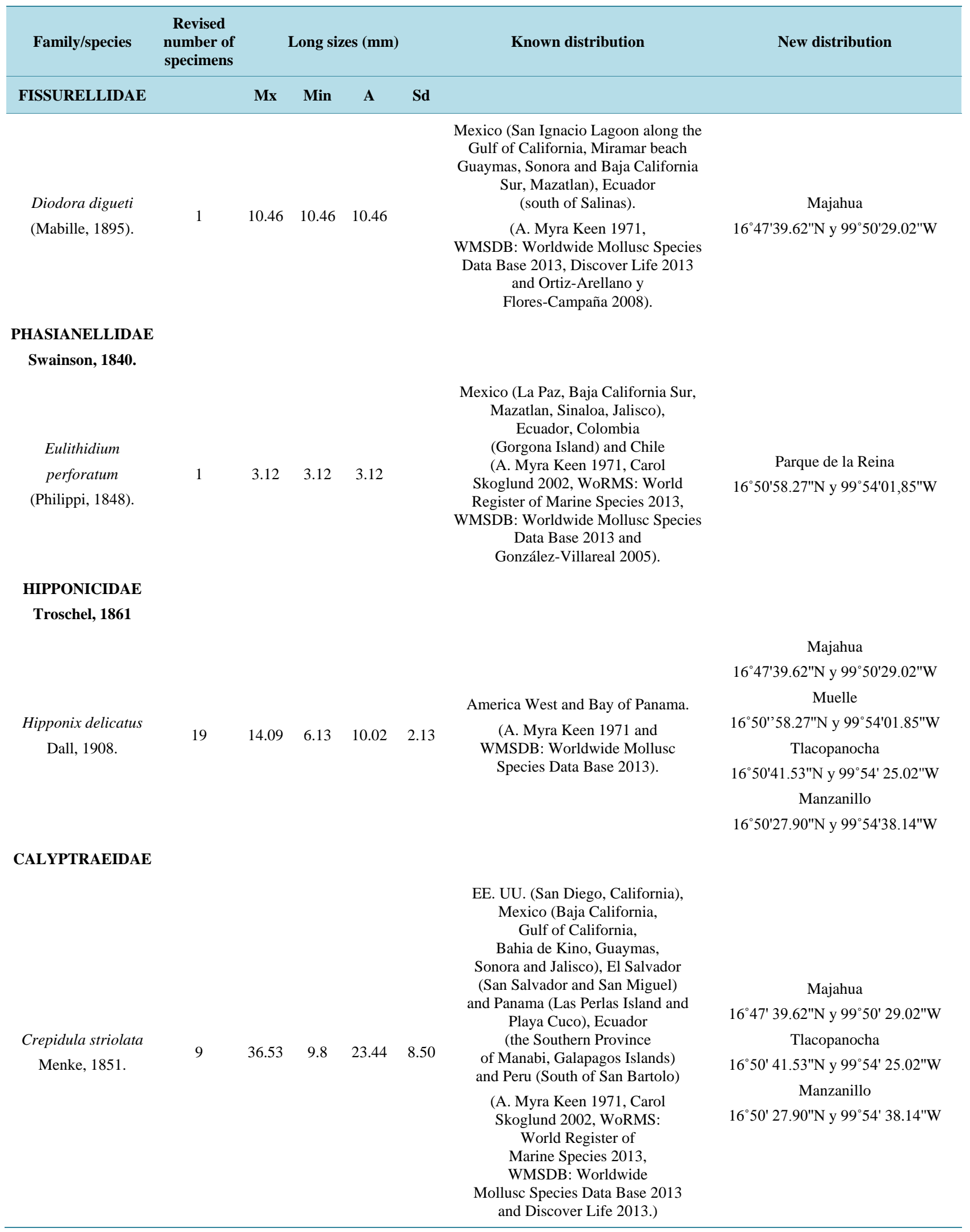




\section{Continued}

\section{COLUMBELLIDAE}

\begin{tabular}{|c|c|c|c|c|}
\hline aureomexicana & 5 & 14.92 & 5.82 & 10.85 \\
\hline
\end{tabular}

Mitrella xenia

(Dall, 1919).

$\begin{aligned} & \text { Costoanachis hilli } \\ & \text { (Pilsbry \& Lowe, }\end{aligned}$
$\begin{gathered}\text { 1932). } \\ \end{gathered}$

Parvanachis gaskoini (Carpenter, 1857).

$\begin{array}{llll}7 & 7.32 & 7.32 & 7.32\end{array}$
Mexico (Isla Cedros and Puertecitos Baja California, Northern Gulf of California to Topolobampo, Sinaloa, Cholla Bay and Miramar Beach, Sonora, Melaque,

Jalisco and Manzanillo, Colima)

(A. Myra Keen 1971, Carol Skoglund 2002,

WMSDB-Worldwide Mollusc Species Data Base 2013 and Discover Life, 2013.)

Mexico (Cabo San Lucas, Baja California Sur and south of Colima), Costa Rica and Panama Province

(A. Myra Keen 1971, Carol Skoglund 2002, WMSDB-Worldwide Mollusc Species Data Base 2013 and Discover Life 2013.

Mexico (North of California,

Baja California Sur, Puerto Peñasco, Sonora and Mazatlán, Sinaloa;) to Nicaragua and Costa Rica.

(A. Myra Keen 1971, WMSDB-Worldwide Mollusc Species Data Base 2013 and

Discover Life 2013)

Mexico (San Luis Gonzaga Bay, Baja California,

Puerto Abreojos,

Baja California Sur,

Sonora Puerto Peñasco, Manzanillo, Colima) and Peru (Callo)

(A. Myra Keen 1971, WMSDB-Worldwide Mollusc Species

Data Base 2013 and Discover Life 2013)

Mexico (Puertecitos, Ensenada, Baja California, Puerto Peñasco Guaymas, Sonora, Bahia Bandera, Nayarit, Colima Manzanillo and Puerto Huatulco, Oaxaca.) To Ecuador (south of Manabi Province)

(A. Myra Keen 1971,

Carol Skoglund 2002 )

\author{
Majahua \\ $16^{\circ} 47^{\prime} 39.62^{\prime \prime N}$ y $99^{\circ} 50^{\prime} 29.02^{\prime \prime} \mathrm{W}$ \\ Parque de la Reina \\ $16^{\circ} 50^{\prime} 58.27 " \mathrm{~N}$ y $99^{\circ} 54^{\prime} 01.85^{\prime \prime} \mathrm{W}$ \\ Muelle \\ $16^{\circ} 50^{\prime \prime} 58.27 " \mathrm{~N}$ y $99^{\circ} 54^{\prime} 01.85^{\prime \prime} \mathrm{W}$ \\ Tlacopanocha \\ $16^{\circ} 50^{\prime} 41.53 " \mathrm{~N}$ y $99^{\circ} 54^{\prime} 25.02^{\prime \prime} \mathrm{W}$ \\ Parque de la Reina \\ $16^{\circ} 50^{\prime} 58.27$ " N y 99 54' 01.85" W \\ Muelle \\ $16^{\circ} 50^{\prime} 58.27 " \mathrm{~N}$ y $99^{\circ} 54^{\prime} 01.85^{\prime \prime} \mathrm{W}$ \\ Tlacopanocha \\ $16^{\circ} 50^{\prime} 41.53^{\prime \prime} \mathrm{N}$ y $99^{\circ} 54^{\prime} 25.02^{\prime \prime} \mathrm{W}$ \\ Manzanillo \\ $16^{\circ} 50^{\prime} 27.90^{\prime \prime} \mathrm{N}$ y $99^{\circ} 54^{\prime} 38.14^{\prime \prime} \mathrm{W}$ \\ La Angosta \\ $16^{\circ} 60^{\prime} 29.86 " \mathrm{~N}$ y $99^{\circ} 54$ ' 55.70"W \\ Pie de la Cuesta \\ $16^{\circ} 52^{\prime} 25.64 " \mathrm{~N}$ y $99^{\circ} 56^{\prime} 34.64 " \mathrm{~W}$
}

Tlacopanocha

$16^{\circ} 50^{\prime} 41.53 " \mathrm{~N}$ y $99^{\circ} 54^{\prime} 25.02^{\prime \prime} \mathrm{W}$

Parque de la Reina

$16^{\circ} 50^{\prime} 58.27^{\prime \prime} \mathrm{N}$ y $99^{\circ} 54^{\prime} 01.85^{\prime \prime} \mathrm{W}$

Muelle

$16^{\circ} 50^{\prime} 58.27^{\prime \prime} \mathrm{N}$ y $99^{\circ} 54^{\prime} 01.85^{\prime} \mathrm{W}$

Majahua

$16^{\circ} 47^{\prime} 39.62 " \mathrm{~N}$ y $99^{\circ} 50^{\prime} 29.02^{\prime \prime} \mathrm{W}$

Keen, 1971. 


\section{Continued}

\section{OLIVELLIDAE}

Troschel, 1969.

Olivella

$\begin{array}{lllll}\text { (Olivella) dama } & 1 & 7.37 & 7.37 & 7.37\end{array}$

(Wood, 1828)

\section{TURRIDAE}

H. Adams \& A.

Adams, 1853.

Crassispira

(Dallspira)

cerithoidea

1

9.26

9.26
Mexico (San Felipe Baja California, Gulf of California, Bahia Magdalena and La Paz, Baja California Sur, Padre Kino and Punta Colorada, Guaymas, Sonora, South Mazatlan, Sinaloa, Jalisco, Revillagigedo Island, Manzanillo, Colima, Oaxaca).

(A. Myra Keen 1971, Carol Skoglund 2002, WoRMS: World Register of Marine Species 2013, WMSDB-Worldwide Mollusc Species Data Base 2013, Discover Life 2013 and Zamorano et al., 2008).
Parque de la Reina

$16^{\circ} 50^{\prime} 58.27 " \mathrm{~N}$ y $99^{\circ} 54^{\prime} 01.85^{\prime \prime} \mathrm{W}$
Mexico (Bahia de los Angeles, Baja California, Mazatlan, Sinaloa and Barra de Navidad, Jalisco, Oaxaca), Costa Rica

(Cocos Island and Punta Arena) and

Panama. (A. Myra Keen 1971; Carol

Skoglund 2002; WMSDB-Worldwide

Mollusc Species Data Base 2013, Discover

(Carpenter, 1857).

Mx.: Maximum, Min.: Minimum, A.: Average and Sd.: Standard deviation.

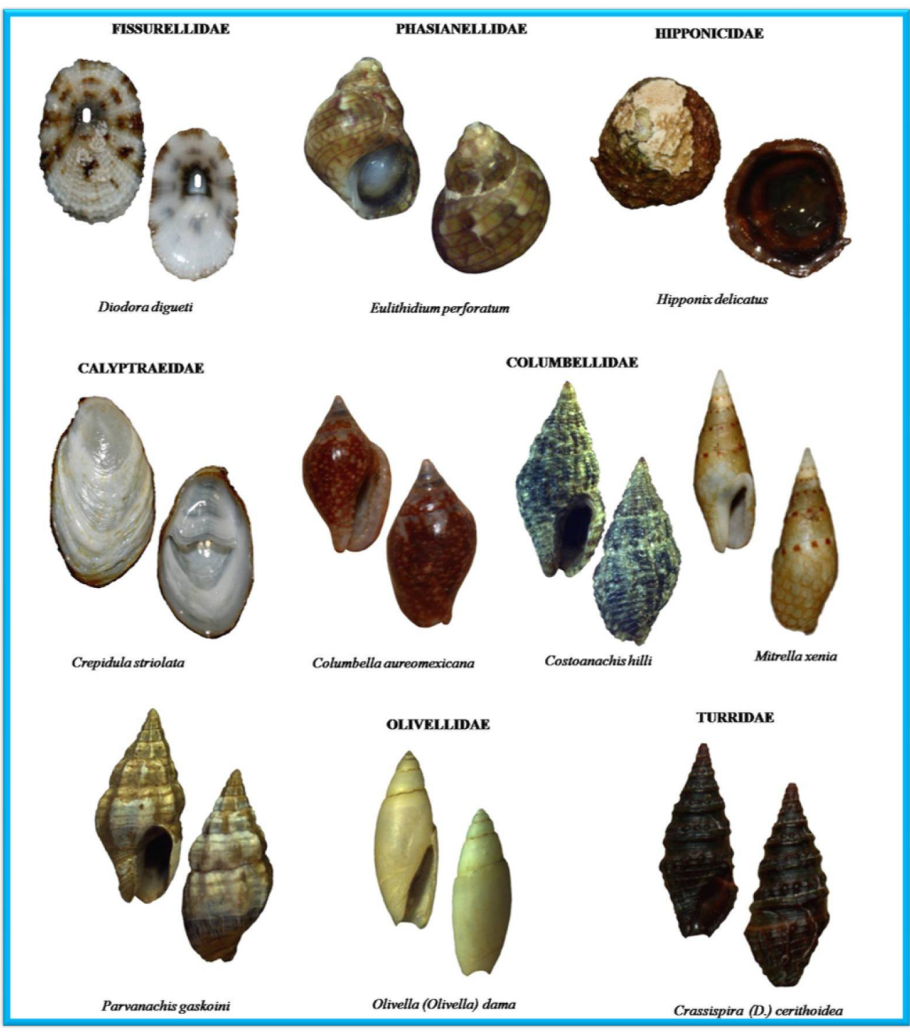

Figure 4. New records for the State of Guerrero. Pictures collection of marine invertebrates, Unidad Académica de Ecología Marina, Universidad Autónoma de Guerrero. Authors: C. Torreblanca-Ramírez, R. Flores-Garza, L. Galeana-Rebolledo y J.C. Cerros-Cornelio. 


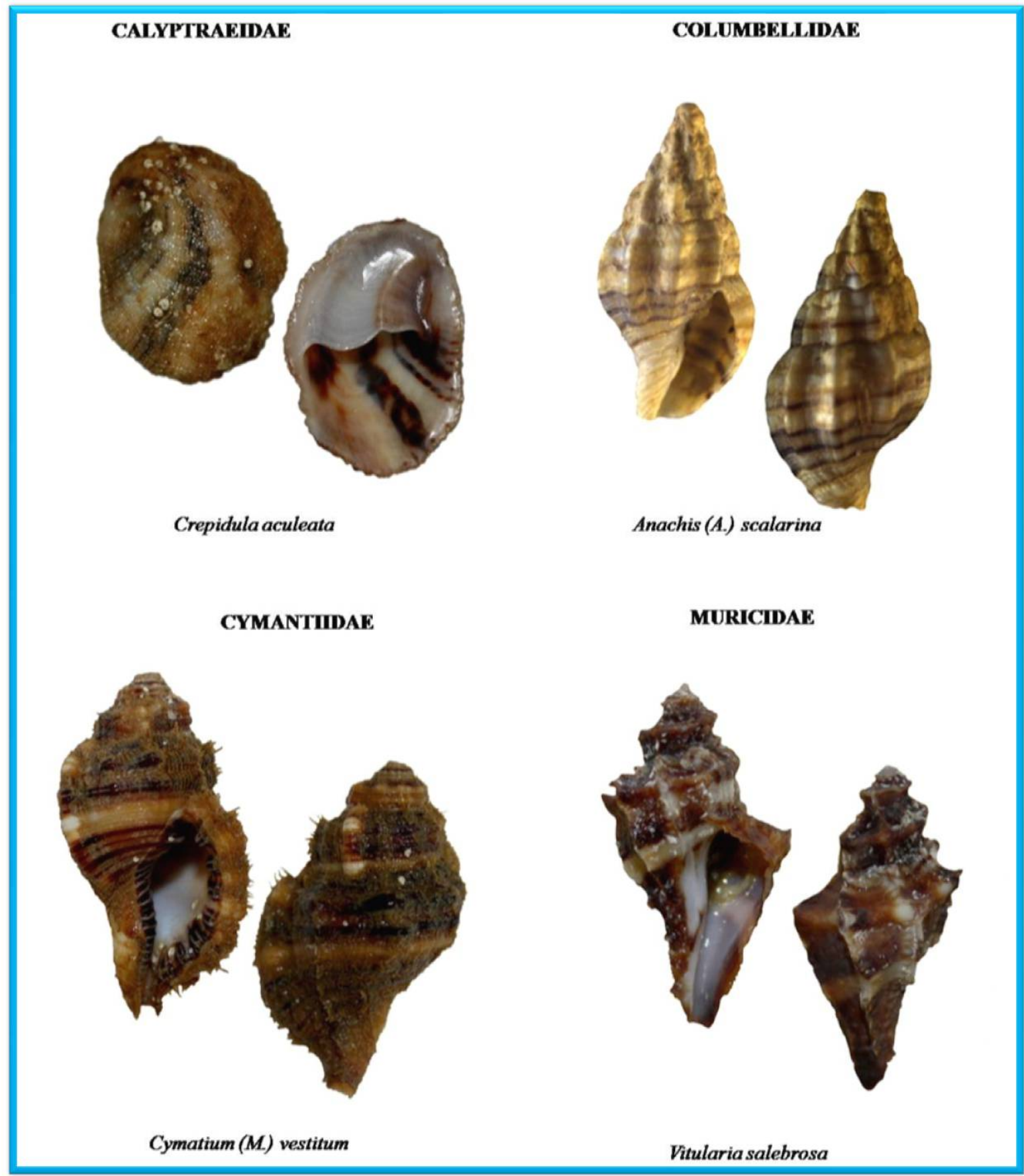

Figure 5. New records for the Marine Priority Region 32. Pictures collection of marine invertebrates, Unidad Académica de Ecología Marina, Universidad Autónoma de Guerrero. Autores: C. Torreblanca-Ramírez, R. Flores-Garza, L. GaleanaRebolledo y J.C. Cerros-Cornelio.

\section{Conclusions}

CONABIO reports that molluscs are a group about which little is known and further research is required in all of Mexico [2]. This contribution reports a significant number of new records for species of class GASTROPODA found on the rocky intertidal zone of the MPR 32, of which several of these records are for Mexico, others for Transitional Mexican Pacific, the state of Guerrero and the MPR 32. With these new records report, we demon- 
Table 5. New records for the Marine Priority Region 32, Guerrero, Mexico, examined material, sizes and distributions prior to this contribution.

\begin{tabular}{|cccccccc}
\hline Family/species & $\begin{array}{c}\text { Revised number } \\
\text { of specimens }\end{array}$ & \multicolumn{2}{c}{ Long sizes $(\mathrm{mm})$} & Known distribution & New distribution \\
\hline CALYPTRAEIIDAE & Mx & Min & A & Sd & \\
\hline
\end{tabular}

Crepidula aculeate

(Gmelin, 1791).
27.99

7.98
EE. UU. (Hawaii, California), Mexico (La Paz, Gulf of California, Baja California Sur, Pacific Coast, Jalisco and Revilagigedo Islands, Manzanillo, Colima, Zihuatanejo), Nicaragua, Costa Rica, Panama (Panama Canal),

Colombia (Malpelo Island ), Ecuador (Guayas Province and Galapagos Islands), Peru (Paita) and Chile (Valparaiso)

(A. Myra Keen 1971, Carol Skoglund 2002, WoRMS-World Register of Marine Species 2013, Discover Life 2013 and Salcedo et al. 1988)

\section{Majahua}

$16^{\circ} 47^{\prime} 39.62 " \mathrm{~N} \mathrm{y}$ $99^{\circ} 50^{\prime} 29.02^{\prime \prime} \mathrm{W}$

Parque de la Reina

$16^{\circ} 50^{\prime} 58,27 " \mathrm{~N}$ y

$99^{\circ} 54^{\prime} 01,85^{\prime \prime} \mathrm{W}$

Muelle

$16^{\circ} 50^{\prime \prime} 58.27 " \mathrm{~N} \mathrm{y}$ $99^{\circ} 54^{\prime} 01.85 " \mathrm{~W}$

Tlacopanocha

$16^{\circ} 50^{\prime} 41.53^{\prime \prime} \mathrm{N}$ y $99^{\circ} 54^{\prime} 25.02 " \mathrm{~W}$

Manzanillo $16^{\circ} 50^{\prime} 27.90 " \mathrm{~N} \mathrm{y}$ $99^{\circ} 54^{\prime} 38.14^{\prime \prime} \mathrm{W}$

\section{CYMANTIIDAE}

Iredale, 1913.

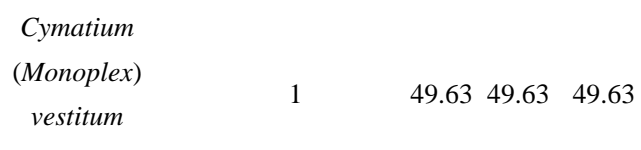

(Hinds, 1844).

\section{MURICIDAE}

Vitularia salebrosa

(King \& Broderip, 1832).
Mexico (Baja California, Gulf of California; Revillagigedo Island Manzanillo, Colima, Zihuatanejo), Clipperton Island, Costa Rica (Cocos, Bahia Culebra), Panama (Venado Island), Colombia (Gorgona Island), Ecuador (Galapagos Islands) and Peru (Paita).

(A. Myra Keen 1971, Carol Skoglund 2002, WoRMS-World Register of Marine Species 2013, WMSDB-Worldwide Mollusc Species Data Base 2013, Discover Life 2013 and Salcedo et al. 1988).

Mexico (Isla Cedros, Baja California, La Paz, Gulf of California, Baja California Sur, Topolobampo, San Carlos, Guaymas, Sonora and Mazatlán, Sinaloa, Roca Partida and Isla Revillagigedo Manzanillo, Colima, Zihuatanejo), Costa Rica (Cocos ) Panama (Coiba Island, East and West of Panama, Gobernadora Island and Montijo Bay) Colombia (Gorgona Island, Malpelo) Ecuador (Manabi Province, Bay Urvina and Galapagos Islands) and Peru (South Talara, Piura) (A. Myra Keen 1971, Carol Skoglund 2002, WoRMS-World Register of Marine Species 2013, WMSDB-Worldwide Mollusc Species Data Base 2013, Discover Life 2013 and Salcedo et al. 1988).

\section{COLUMBELLIDAE}

\section{Anachis \\ (Anachis)}

scalarina

2
$6.15 \quad 5.18 \quad 5.66$
Mexico (Guaymas, Sonora and Mazatlán, Sinaloa, Zihuatanejo), Panama (Venado Island) 0.68
(A. Myra Keen 1971, WMSDB-Worldwide Mollusc Species Data Base 2013, Discover Life 2013 and Salcedo et al. 1988.)
Ploaya Majahua

$16^{\circ} 47^{\prime} 39.62 " \mathrm{~N} \mathrm{y}$ $99^{\circ} 50^{\prime} 29.02^{\prime \prime} \mathrm{W}$

Playa Manzanillo $16^{\circ} 50^{\prime} 27.90^{\prime \prime} \mathrm{N} \mathrm{y}$ $99^{\circ} 54^{\prime} 38.14 " \mathrm{~W}$

(Sowerby, 1832)

Mx.: Maximum, Min.: Minimum, A.: Average and Sd.: Standard deviation. 
strate the need to focus research efforts on the study of marine diversity. This has been noted by CONABIO, especially in regions that have been identified as priorities for conservation and use of biodiversity in Mexico.

This report increases the biodiversity information for malacological fauna on the local and regional scales and establishes a basis for future investigations of marine molluscs.

\section{References}

[1] Wilkinson, T., Wiken, E., Bezaury, C.J., Hourigan, T., Agardy, T., Herrmann, H., Janishevski, L., Madden C., Morgan, L. and Padilla, M. (2009) Ecorregiones marinas de América del Norte. Comisión para la Cooperación Ambiental, Montreal.

[2] Arriaga, C.L., Vázquez, D.E., González, C.J., Jiménez, R.R., Muñoz, L.E. and Aguilar S.V. (1998) Regiones marinas prioritarias de México. Comisión Nacional para el Conocimiento y uso de la Biodiversidad, México.

[3] López, de la F.A. and Urcuyo, R.J. (2009) Moluscos de Nicaragua II, Gastrópodos. Marena-Araucaria, UCA, Managua.

[4] Tucker, J.K. and Tenorio, M.J. (2009) Systematic Classification of Recent and Fossil Conoidean Gastropods. Conchbooks, Hackenheim.

[5] Tenorio, M.J, Tucker, J.K. y Chaney, H.W. (2012) The Families Conilithidae and Conidae. The Cones of the Eastern Pacific. In: Poppe y Groh, G.T., Ed., A Conchology Iconography, Conchbooks, Hackenheim.

[6] Keen, A.M. (1971) Sea Shells of Tropical West America. Stanford University Press. Palo Alto.

[7] Brusca, R.C. (1980) Common Intertidal Invertebrates of the Gulf of California. University of Arizona Press, Tucson.

[8] Skoglund, C. (2002) Panamic Province Molluscan Literature Additions and Changes from 1971 through 2001, III Gastropoda. The Festivus, 33, 1-286.

[9] Holguín, O.E. and González, A.C. (1989) Moluscos de la franja costera del Estado de Oaxaca, México. Dirección de Bibliotecas y Publicaciones, Instituto Politécnico Nacional, Mexico City.

[10] Reguero, M. and García-Cubas, A. (1989) Moluscos de la Plataforma Continental de Nayarit: Sistemática y Ecología (cuatro campañas oceanográficas). Anales del Instituto de Ciencias del Mar y Limnología, Universidad Nacional Autónoma de México, 16, 33-58.

[11] Holguín-Quiñones, O.E. and González-Pedraza, A.C. (1994) Moluscos de la franja costera de Michoacán, Colima y Jalisco, México. Dirección de Bibliotecas y Publicaciones, Instituto Politécnico Nacional, Mexico City.

[12] Román, R., Cruz, F.M. and Ibáñez, A.L. (1991) Observaciones ecológicas de los moluscos de la zona intermareal de la bahía de Chamela, Jalisco, México. Anales del Instituto de Biología, Universidad Nacional. Autónoma de México, Serie Zoología, 62, 17-32.

[13] Ríos-Jara, E., Pérez, M., Lizárraga, L. and Michel-Morfín, J.E. (1996) Nuevos registros de la plataforma continental de Jalisco y Colima, México. Ciencias Marinas, 22, 347-359. http://www.redalyc.org/pdf/480/48022306.pdf

[14] Landa-Jaime, V. and Arciniega-Flores, J. (1998) Macromoluscos bentónicos de fondos blandos de la plataforma continental de Jalisco y Colima, México. Ciencias Marinas, 24, 155-167.

[15] Esqueda, M.C., Ríos-Jara, E., Michel-Morfín, J.E. and Landa-Jaime, V. (2000) The Vertical Distribution and Abundance of gastropods and Bivalves from Rocky Beaches of Cuastecomate Bay, Jalisco. Revista de Biología Tropical, 48, 765-775.

[16] Olabarría, C. and Vega, C. (2000) Extensión del ámbito geográfico de algunas especies de moluscos marinos en el estado de Sinaloa, México. Anales del instituto de Biología, Universidad Nacional Autónoma de México, Serie Zoológica, 71, 93-98. http://www.biblioteca.org.ar/libros/91823.pdf

[17] Villarroel, M.M., Magaña, A., Gómez, B., Del Río, O., Lucio, J. and Sánchez, J. (2000) Diversidad de moluscos en el litoral rocoso de Michoacán, México. Mexicoa, 2, 54-63.

[18] González-Villareal, L. M. (2005) Guía ilustrada de los gasterópodos marinos de la bahía de Tenacatita, Jalisco, México. Scientia-CUCBA, 7, 1-84.

[19] Landa-Jaime, V., Cruz-Urzua, M., Michel-Morfín, J. E., Archiniega-Flores, J., Flores-Vargas, R. and Amezcua, C. (2007) Guía ilustrada para la identificación de moluscos intermareales y de arrecifes en la Bahía de Tenacatita, Jalisco. In: Ríos-Jara, E., Esqueda-González, M.C. and Galván-Villas, C.,M., Eds., Estudios sobre la Malacología y Conquiliología en México, Universidad de Guadalajara, Guadalajara, 63-64.

[20] Vega, C., Olabarria, C. and Carballo, J.L. (2008) Variación espacio-temporal de moluscos y macroalgas en sustratos rocosos intermareales en la bahía de Mazatlán. Ciencia y Mar, XII, 3-16. http://www.umar.mx/revistas/34/MOLUSCOS.pdf

[21] Zamorano, P., Barrientos-Luján, N.A. and Ramírez-Luna, S. (2008) Malacofauna del infralitoral rocoso de Agua 
Blanca, Santa Elena Cozoaltepec, Oaxaca. Ciencia y Mar, XII, 19-33.

http://www.umar.mx/revistas/36/malacofauna.pdf

[22] Ortíz-Arellano, M.A. and Flores-Campaña, L.M. (2008) Catálogo descriptivo e ilustrado de los moluscos de la zona intermareal de las Islas de Navachiste, Sinaloa, México. Universidad Autónoma de Sinaloa y Gobierno del Estado de Sinaloa-Consejo Nacional de Ciencias y Tecnología, Mazatlán.

[23] Flores-Rodríguez, P., Barba-Marino, F., Flores-Garza, R., García-Ibáñez, S. and Arana-Salvador, D.G. (2010) Análisis de la comunidad de moluscos del mesolitoral rocoso en playa Corralero, Oaxaca, México. In: Rangel, J.L., Gamboa, J., Arriaga, S.L. and Contreras W.M., Eds., Perspectiva en malacología mexicana, Universidad Juárez Autónoma de Tabasco, Villahermosa, pp.

[24] Landa, J.V. (2013) Bases ecológicas de los caracoles marinos del Género Conus asociados al arrecife coralino de Tenacatita, Jalisco, México. Tesis Doctoral, Universidad Autónoma de Nayarit en Nayarit, (City location) México.

[25] Villalpando, C.E. (1986). Diversidad y zonación de moluscos de superficie rocosa, Isla Roqueta, Acapulco, Guerrero. Tesis de Licenciatura, Universidad Nacional Autónoma de México, Mexico City.

[26] Salcedo, M.S., Green, G., Gamboa, C.A. and Gómez, P. (1988) Inventario de macroalgas y macroinvertebrados béntico, presentes en áreas rocosas de Zihuatanejo, Gro, Méx. Instituto de Ciencias Del Mar y Limnologia, Universidad Nacional Autónoma de México, Mexico City, 73-96.

[27] Delgado, V.H. (1989) Estudio sistemático y aspectos ecológicos de gasterópodos de la facie rocosa de la bahía de Acapulco, Guerrero, México. Tesis de Licenciatura, Escuela Superior de Ecología Marina, Universidad Autónoma de Guerrero, México.

[28] García, L.J.A. (1994) Fauna malacológica de acompañamiento del caracol Purpura pansa Gould 1853 en la zona mesolitoral de la Isla Roqueta, Acapulco, Guerrero, México. Tesis de Licenciatura, Escuela Superior de Ecología Marina, Universidad Autónoma de Guerrero, México.

[29] Flores-Rodríguez, P., Flores-Garza, R., García-Ibáñez, S. and Valdés-González, A. (2003) Riqueza y diversidad de la malacofauna del mesolitoral rocoso de la Isla la Roqueta, Acapulco, Guerrero, México. Ciencia, Revista de Investigación Científica, 11, 5-14.

[30] Flores, R.P. (2004) Estructura de la comunidad de moluscos del mesolitoral superior en las playas de facie rocosa del Estado de Guerrero, México. Tesis doctoral, Facultad de Ciencias Biológicas, Universidad Autónoma de Nuevo León, México.

[31] Flores-Rodríguez, P., Flores-Garza, R., García-Ibáñez, S. and Valdés-González, A. (2007) Variación en la diversidad malacológica del mesolitoral rocoso en Playa Troncones La Unión, Guerrero, México. Revista Mexicana de Biodiversidad, 78, 33S-40S.

[32] Barba-Marino, F., Flores-Rodríguez, P., Flores-Garza, R., García-Ibáñez, S. and Arana-Salvador, D.G. (2010) Biodiversidad y zonificación de la comunidad de moluscos, que habita el sustrato rocoso en dos sitios con distinta acción del oleaje, en la Isla "La Roqueta” Acapulco, Guerrero, México. En: Rangel, J.L., Gamboa, J., Arriaga, S.L. and Contreras, W.M., Eds., Perspectiva en malacología mexicana, Universidad Juárez Autónoma de Tabasco, México.

[33] Torreblanca, R.C. (2010) Análisis de la diversidad y estructura de la comunidad de moluscos del mesolitoral rocoso de Acapulco, Gro. Tesis de Licenciatura, Unidad Académica de Ecología Marina, Universidad Autónoma de Guerrero, México.

[34] Flores-Garza, R., Torreblanca-Ramírez, C., Flores-Rodríguez, P., García-Ibáñez, S., Galeana-Rebolledo, L., Valdés-González, A. and Rojas-Herrera, A.A. (2011) Mollusca Community from a Rocky Intertidal Zone in Acapulco, México. Biodiversity, 12, 144-153. http://dx.doi.org/10.1080/14888386.2011.625520

[35] Pedro, F., Rafael, F., Sergio, G., Arcadio, V., Juan, V., Enedina, S., Lizeth, G. and Carmina, T. (2012) Mollusk Species Richness on the Rocky Shores of the State of Guerrero, Mexico, as Affected by Rains and Their Geographical Distribution. Natural Resources, 3, 248-260. http://www.scirp.org/journal/PaperInformation.aspx?paperID=26293

[36] Torreblanca, R.C., Flores, G.R., Flores, R.P., García, I.S., Valdés, G.A. and Galeana, R.L. (2012) Gastrópodos del intermareal rocoso en Tlacopanocha, Acapulco, México. Tlamati Sabiduría, 4, 47-57.

[37] Torreblanca-Ramírez, C., Flores-Garza, R., Flores-Rodríguez, P., García-Ibáñez, S. and Galeana-Rebolledo, L. (2012) Riqueza, composición y diversidad de la comunidad de moluscos asociada al sustrato rocoso intermareal de playa Parque de la Reina, Acapulco, Guerrero, México. Revista de Biología y Oceanografía, 47, 283-294.

http://www.revbiolmar.cl/index.php?option=com_content\&view=article\&id=681\%3Avolumen-47-2-indice\&catid=969 \&Itemid=68\&lang=es

[38] Mottana, A., Crespi, R. and Liborio, G. (1980) Guía de minerales y rocas, segunda edición. Grijalbo, Barcelona.

[39] Flores-Garza, R., Galeana-Rebolledo, L., Reyes-Gómez, A., García-Ibáñez, S., Torreblanca-Ramírez, C., Flores-Rodríguez, P. and Valdés González, A. (2012) Polyplacophora Species Richness, Composition and Distribution of Its Community Associated with the Intertidal Rocky Substrate in the Marine Priority Region No. 32 in Guerrero, Mexico. Open Jour- 
nal of Ecology, 2, 192-201. http://www.scirp.org/journal/PaperInformation.aspx?paperID=24567\#.U0bdzvl5OX4

[40] WoRMS-World Register of Marine Species (2013) http://www.marinespecies.org/index.php

[41] WMSDB-Worldwide Mllusc Species Data Base (2013)

http://www.bagniliggia.it/WMSD/HtmSpecies/1933700420.htm

[42] Discover Life (2013) http://www.discoverlife.org/mp/20q 
Scientific Research Publishing (SCIRP) is one of the largest Open Access journal publishers. It is currently publishing more than 200 open access, online, peer-reviewed journals covering a wide range of academic disciplines. SCIRP serves the worldwide academic communities and contributes to the progress and application of science with its publication.

Other selected journals from SCIRP are listed as below. Submit your manuscript to us via either submit@scirp.org or Online Submission Portal.
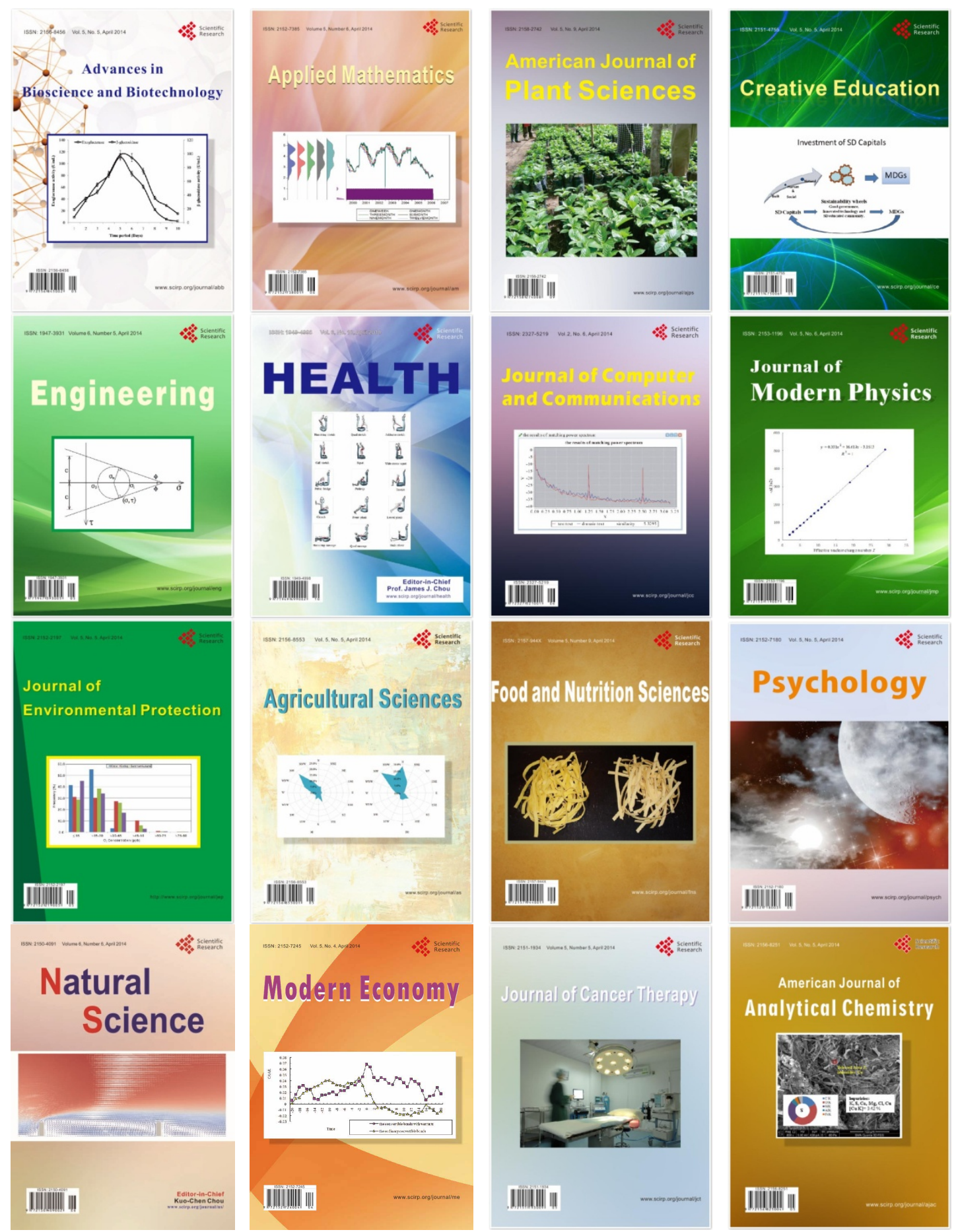